FZJ-IKP(TH)-2004-01, HISKP-TH-04/03

\title{
The role of the nucleon recoil in low-energy meson-nucleus reactions
}

\author{
V. Baru ${ }^{1,2}$, C. Hanhart ${ }^{1}$, A.E. Kudryavtsev², and U.-G. Meißner ${ }^{1,3}$ \\ ${ }^{1}$ Institut für Kernphysik, Forschungszentrum Jülich GmbH, \\ D-52425 Jülich, Germany \\ ${ }^{2}$ Institute of Theoretical and Experimental Physics, \\ 117259, B. Cheremushkinskaya 25, Moscow, Russia \\ ${ }^{3}$ Helmholtz-Institut für Strahlen- und Kernphysik (Theorie), Universität Bonn \\ Nußallee 14-16, D-53115 Bonn, Germany
}

\begin{abstract}
The role of the nucleon recoil corrections in low-energy meson-nucleus interactions is examined. We demonstrate explicitly when calculations within the static approximation are justified and when the recoil terms need to be kept explictly in the propagators, depending on whether the S-wave two-nucleon intermediate state is Pauli blocked or not, while the meson is in flight. While the effect is studied in detail for $\pi d$ scattering only, a large class of other reactions is discussed for which the findings are relevant as well.
\end{abstract}

\section{Introduction}

Low-energy meson-nucleon reactions are of great theoretical interest for they are one of the best tools to deepen our understanding of the nuclear many-body problem. The production and scattering of the lightest member of the Goldstone nonet, i.e. the pion, on nuclei is the subject of special experimental and theoretical interest since they allow to test predictions of chiral perturbation theory and - within this scheme - quantify the effect of isospin violation in the strong $\pi N$ interaction. In addition, pion-nucleus reactions can be used to get information on the elementary pion-neutron interactions. Detailed knowledge of the latter is important to fix the isoscalar pion-nucleon scattering length. The calculation of the production and scattering processes for heavier mesons on nuclei is more difficult but not less interesting. For instance, the reactions involving the $\eta$ meson can be used to explore the possibility of the formation 
of the $\eta$-nucleus bound state, whereas the $K d$-scattering allows to extend our knowledge about the strange sector.

It is well known that rescattering effects, where the intermediate meson being scattered on one nucleon of the nucleus then rescatters on another one, are potentially large and need to be evaluated in a controlled way in order to, for example, extract information on the the neutron amplitudes. How to do this within an effective field theory Weinberg described in one of his classic papers [1]. In this paper nucleons are treated rigorously as static, as long as the diagram is two-nucleon irreducible, leading to largely simplified nuclear matrix elements. This framework has been subsequently extended to higher orders and consistent wave functions based on chiral nuclear effective field theory have been used, see $[2,3]$.

In this note we investigate under what circumstances the static approximation is justified and also identify reactions where the recoil corrections are to play a significant role. On the example of the calculation of the $\pi d$ scattering length we show that cancellations amongst different one-body and two-body amplitudes have to occur in order to make the Pauli principle also hold for two-nucleon states while there is a pion in flight simultaneously. Similar arguments were recently presented by Rekalo et al. [4] for neutral pion photoand electroproduction on the deuteron at low energies. However, these authors argue that in case of a Pauli forbidden intermediate state the pion rescattering contribution has to be canceled completely in order to allow the nucleons to obey the Pauli principle. In this paper we critically reexamine this claim. Especially we find that it is not the full rescattering amplitude that vanishes in case of the Pauli forbidden S-wave intermediate two-nucleon state, but only the part stemming from the nucleon recoils, leaving the static exchange as a good approximation to the full result. Note that the interference pattern discussed was already observed numerically in the phenomenological approach of Refs. [5,6]. In addition, we will show that, at least for $\pi d$ scattering near threshold, for cases where the S-wave intermediate two-nucleon state is Pauli allowed, the net effect of the (then important) recoil corrections is that the corresponding rescattering contributions (i.e. the static term + the corrections occuring due to the finite nucleon mass) are numerically irrelevant.

Historically the presence of sizable cancellation in calculation for the $\pi d$ scattering length was raised first in the papers by Kolybasov et al. [7] and independently by Fäldt [8] where it was claimed that the naive static term is a good approximation for rescattering effects (see also discussion in Ref. [9]). However, the effect in Ref. [8] was traced to a quite unnatural numerical cancellation of the diagrams shown in Fig. 1 b) and c) with the corresponding diagrams where the intermediate nucleons are rescattered off each other. Moreover in Ref. [8] rescattering effects are approximated by the static term for both types of the S-wave $\pi N$-potential, i.e. for isoscalar and isovector. This is indeed correct 


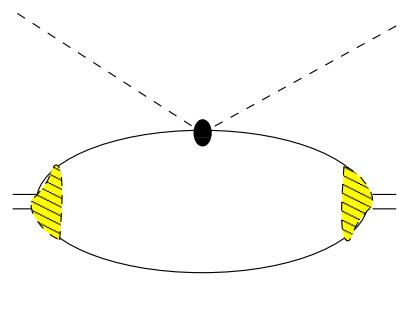

a)

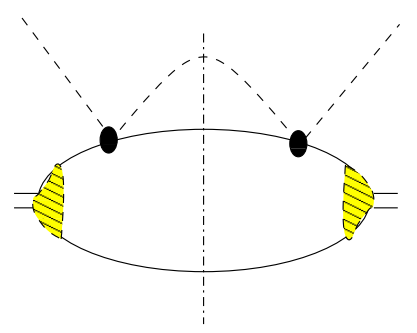

b)

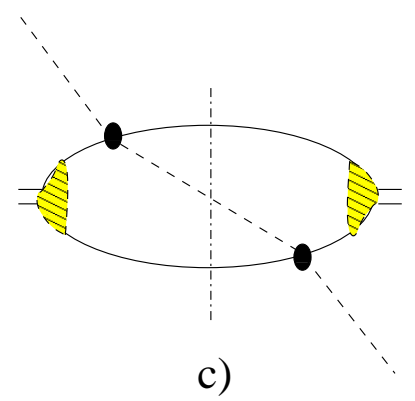

c)

Fig. 1. Typical Feynman diagrams for $\pi d$ scattering; shown are one-body terms (diagram a) and b), as well as the corresponding rescattering contribution c) ). Solid black dots stand for the $\pi N$ interaction, whereas the hatched area shows the deuteron wave function. Crossed terms (where the external pion lines are interchanged) are not shown explicitly.

for the isovector $\pi N$-interaction but not for the isoscalar one as we will prove below. Due to the smallness of the isoscalar $\pi N$-scattering length the wrong interpretation of rescattering effects in this case does not affect the full result of the calculation of the $\pi d$-scattering length, however, for other processes where this term is not small one would get the incorrect result.

Note that arguments based on the Pauli principle for the intermediate nucleons are very general and therefore can be applied for different processes with any intermediate meson exchanges. Several examples will be discussed in sec. 3 .

\section{$2 \quad$ Pauli principle and the $\pi d$ scattering length}

To be concrete, this discussion will be carried out for the reaction $\pi d \rightarrow \pi d$ and we will restrict ourselves to a rather simplified $\pi N$ interaction that, however, allows us to address all relevant issues.

In Fig. 1 we show typical single nucleon diagrams a) and b) as well as the corresponding two-nucleon contribution c). We will concentrate on the isolation of the one-body (isoscalar) amplitude (as it would be also measured in a $\pi N$ scattering experiment or extracted from a partial-wave analysis) from that related to nuclear effects.

In general, two-nucleon states in any subsystem have to obey the Pauli principle. Thus, when looking at diagram c), where the relevant intermediate state is marked by a perpendicular line, the Pauli principle demands the two nucleons to show a particular behavior under their exchange. However, if we would exchange the two nucleons in the intermediate state of diagram c) we come automatically to diagram b). Thus, we should expect some destructive interference between b) and c) if the intermediate state is forbidden by the Pauli 
principle and a constructive one if the intermediate state is allowed.

The interference pattern depends on the particular structure of the $\pi N \rightarrow \pi N$ transition potential. At the threshold it may be written as (the factor $1 / \sqrt{2}$ was introduced for convenience since this way we will get results symmetric in $g_{+}$and $g_{-}$)

$$
V_{\pi N}^{b a}=\delta^{b a} g_{+}+\frac{i}{\sqrt{2}} \epsilon^{a b c} \tau^{c} g_{-},
$$

where the strength of the isoscalar (isovector) interaction is denoted by $g_{+}\left(g_{-}\right)$ and $a(b)$ is the isospin index of the initial (final) pion. For our study we assume both $g_{+}$and $g_{-}$as constant. The conclusions do not change by this simplification, but the formulas simplify largely. As was shown by Weinberg [10], Tomozawa [11] and others, $\left(g_{+} / g_{-}\right) \sim\left(m_{\pi} / \Lambda\right)$, where $\Lambda$ denotes the typical hadronic scale of order $1 \mathrm{GeV}$ and $m_{\pi}$ is the pion mass. Thus, in $\pi d$ scattering the isoscalar rescattering contribution is largely suppressed. However, for illustration we will keep both $g_{+}$and $g_{-}$in the calculation.

Lets us now analyze more closely the structure of the matrix element corresponding to diagram 1c). The deuteron is an isoscalar. Thus, when a scalar (vector) operator in isospin space operates on this state, the resulting twonucleon state is in an isoscalar (isovector) state. At threshold the $\pi N$ interaction is spin and momentum-independent and therefore the two-nucleon state after the $\pi N$ interaction is in both scenarios in a spin triplet $S$-wave state. The Pauli principle allows this only for isoscalars and thus, if the $\pi N$ interaction is given by the $g_{+}$term, the contributions of diagrams c) and b) to the $\pi d$ scattering length due to $N N$ recoil should interfere constructively and, if it is given by the $g_{-}$term, they should interfere destructively.

Within our simplified model the $\pi d$ scattering length is given by

$$
a=a_{a}+a_{b}^{+}+a_{b}^{-}+a_{c}^{+}+a_{c}^{-},
$$

where the superscripts denote the isospin structure of the $\pi N$-interaction and the subscripts refer to the diagrams as shown in Fig. 1. Since the deuteron is an isoscalar state, only $g_{+}$contributes to diagram a), and we get

$$
a_{a}=\frac{2 g_{+}}{4 \pi\left(1+m_{\pi} /\left(2 M_{N}\right)\right)} .
$$

We also give the expressions for diagram b)

$$
a_{b}^{ \pm}=g_{ \pm}^{2} \xi \int d^{3} p d^{3} q \Psi(\vec{p})^{\dagger} \frac{1}{\vec{q}^{2}+\rho} \Psi(\vec{p})
$$

and for diagram c)

$$
a_{c}^{ \pm}= \pm g_{ \pm}^{2} \xi \int d^{3} p d^{3} q \Psi(\vec{p}-\vec{q})^{\dagger} \frac{1}{\vec{q}^{2}+\rho} \Psi(\vec{p})
$$


where $\Psi$ denotes the deuteron wave function and $\mathrm{p}(\mathrm{q})$ is the relative nucleon momentum before the $\pi N$ interaction (the intemediate pion momentum). Furthermore, $\xi=\left[16 \pi^{4}\left(1+m_{\pi} /\left(2 M_{N}\right)\right)\right]^{-1}, \rho=\omega\left(2 \epsilon+\left(\vec{p}^{2}+(\vec{p}-\vec{q})^{2}\right) / M_{N}\right)$, with $\epsilon$ and $\omega$ for the deuteron binding energy and the pion energy, respectively, and $M_{N}$ is the nucleon mass. To come to these expressions terms of order $\rho^{2}(\rho)$ were dropped in the denominator (numerator), since they lead only to the complication of the formulae but are irrelevant for this study. The piece linear in $\rho$ that appears in the denominators is the recoil correction of interest. In Ref. [1] as well as many multiple scattering formalisms (see Ref. [9] and references therein) the structure given in Eq. (5) with $\rho=0$ is given for the rescattering contribution. However, when straightforwardly evaluated the inclusion of $\rho$ decreases the value of $a_{c}$ approximately by a factor of 2 [5] (see also Ref. [12] where the recoil corrections were estimated for the first time and found to be important). The reason for this relatively large effect of the recoil corrections on the amplitude is the proximity of a three-body singularity: the fact that for $\epsilon=0$ the intermediate states can become real leads to a contribution non-analytic in the nucleon mass that can not be simply dropped.

The goal of our study is two-fold: we want to split the $\pi d$ amplitude into its contribution from the $\pi N$ amplitude and the nuclear corrections - the socalled rescattering contributions - and secondly we want to understand the role of the nucleon recoil in the latter. The former issue was previously addressed within chiral perturbation theory in Refs. [1,2,3], but this separation is much more transparent in our simplified approach and thus allows to discuss the various contributions more directly. Thus, after adding and subtracting appropriate terms, we decompose the expression for the $\pi d$ scattering length in the following way

$$
a=a^{(1-b o d y)}+a_{L O}^{(\text {static })}+a^{(\text {recoil })}+a_{N L O}^{(\text {static })} .
$$

Here the one-body piece is given by

$$
a^{(1-b o d y)}=\frac{2 g_{+}}{4 \pi\left(1+m_{\pi} /\left(2 M_{N}\right)\right)}+\left(g_{+}^{2}+g_{-}^{2}\right) \xi \int d^{3} q \frac{1}{\vec{q}^{2}+\tilde{\rho}}
$$

where $\tilde{\rho}=\omega \vec{q}^{2} / M_{N}$ and a particular regularization prescription is to be given to render the loop integral finite (see e.g. Ref. [13]). Note that within our model the full expression given in Eq. (7) is the expression for the isoscalar $\pi N$ scattering length. It is therefore this piece that we need to isolate, if we want to extract single nucleon amplitudes from nuclear reactions. The rescattering contribution (or nuclear corrections) we split without any further approximations into the leading order static piece given in Ref. [1]

$$
a_{L O}^{(\text {static })}=\left(g_{+}^{2}-g_{-}^{2}\right) I_{0}
$$


with

$$
I_{0}=\xi \int d^{3} p d^{3} q \Psi(\vec{p}-\vec{q})^{\dagger} \frac{1}{\vec{q}^{2}} \Psi(\vec{p}),
$$

and the corrections occuring due to the finite nucleon mass which are again separated on two parts, namely the 3-body singularity correction (or the recoil term) which is not analytic in $\omega_{\pi} / M_{N}$ and the term $a_{N L O}^{(\text {static })}$ which is regular in $\omega_{\pi} / M_{N}$. These corrections can be expressed as

$$
a^{(\text {recoil })}=g_{+}^{2} I_{+}+g_{-}^{2} I_{-}
$$

with

$$
I_{ \pm}=\frac{\xi}{2} \int d^{3} p d^{3} q|\Psi(\vec{p}) \pm \Psi(\vec{p}-\vec{q})|^{2}\left(\frac{1}{\vec{q}^{2}+\rho}-\frac{1}{\vec{q}^{2}+\tilde{\rho}}\right)
$$

where we used the symmetry of $I_{ \pm}$to replace $\Psi(\vec{p})$ by $\frac{1}{2}(\Psi(\vec{p}) \pm \Psi(\vec{p}-\vec{q}))$ and

$$
a_{N L O}^{(\text {static })}=\left(g_{+}^{2}-g_{-}^{2}\right) \Delta I
$$

with

$$
\Delta I=\xi \int d^{3} p d^{3} q \Psi(\vec{p}-\vec{q})^{\dagger}\left(\frac{1}{\vec{q}^{2}+\tilde{\rho}}-\frac{1}{\vec{q}^{2}}\right) \Psi(p) \simeq-\frac{m_{\pi}}{M_{N}} I_{0}
$$

Note that $a^{(\text {static })}, a^{(\text {recoil })}$ and $a_{N L O}^{(\text {static })}$ are finite.

We numerically evaluated $I_{ \pm}$and $I_{0}$ using the deuteron wave functions from the Bonn potential [14] and found

$$
I_{+}=-0.88, \text { and } I_{-}=-0.19,
$$

both given in units of $I_{0}$. We checked that the numbers do not depend on the type of the deuteron wave function used. The results reflect the interference pattern discussed above, i.e. that the 3 -body correction due to the nucleon recoil is much smaller in the case when the $\mathrm{S}$-wave $N N$ intermediate state is forbidden by the Pauli principle compared to that when it is allowed $\left(I_{-} \ll I_{+}\right)$. To make it even more clear that the interference pattern in $I_{ \pm}$indeed reflects what is demanded by the Pauli principle let us express the integrals explicitly in terms of the two-nucleon relative momentum, $\vec{p}^{\prime}=\vec{p}-\vec{q} / 2$, for the $N N$ intermediate state. Then the first term of the integrant in Eq. (11) reads

$$
\Psi\left(\vec{p}^{\prime}+\frac{1}{2} \vec{q}\right) \pm \Psi\left(\vec{p}^{\prime}-\frac{1}{2} \vec{q}\right)
$$


For the upper (lower) sign this combination is symmetric (antisymmetric) under the transformation ${ }^{1} \vec{p}^{\prime} \rightarrow-\vec{p}^{\prime}$. Thus, in case of $I_{+}\left(I_{-}\right)$this term projects on the Pauli allowed even (odd) two-nucleon spin triplet states in the $N N \pi$ intermediate state - a statement that holds for both the imaginary part as well as the real part!

Note also that the contributions of diagrams $1 \mathrm{~b}$ ) and c) to the imaginary part of the $\pi d$ scattering length (e.g. due to charge exchange process) can appear only from the recoil term, i.e. from $I_{+}\left(I_{-}\right)$. In case of the calculation of the imaginary part of $I_{+}\left(I_{-}\right)$one has to integrate over the momenta in the narrow region restricted by the 3 -body phase space, i.e. over the region where the contribution of the $\mathrm{P}$-wave $N N$ state is small. This leads to a rather small value of $\operatorname{Im} I_{-}$.

We stress that $I_{+}$is large and negative. Therefore when adding the terms proportional to $g_{+}^{2}$ in $a^{(\text {recoil })}$ and $a^{(\text {static })}$, they largely cancel. On the other hand, $I_{-}$is much smaller than $I_{0}$ and therefore the nuclear contributions to the $\pi d$ scattering amplitude proportional to $g_{-}^{2}$ are basically given by $a^{(\text {static })}$. Thus, we find that if the S-wave two-nucleon intermediate state that occurs while the pion is in flight is allowed by the Pauli principle, the net effect of the rescattering contribution is quite small. On the other hand, if the S-wave twonucleon intermediate state is Pauli forbidden, the rescattering effects are large, however, can be well approximated by a static exchange. The corrections to this are found to be of order $15-20 \%$ of the static term. However, one should also not forget about the NLO correction to the static term. This correction is not related with the Pauli principle and it is regular in $\omega / M_{N}$. Moreover, in distinction from the 3-body recoil correction, which is only weakly dependent on the mass of the exchanged meson, the NLO correction to the static term linearly depends on the mass. It turned out that for the $\pi d$ scattering the integral $\Delta I$ is equal to -0.15 (in the same units), thus making the conclusion about the role of rescattering effects presented above even stronger. Indeed, this term interferes destructively with $I_{-}$(constructively with $I_{+}$) resulting in the total correction to the static term (Eq. (8)) to be equal to

$$
a^{(\text {recoil })}+a_{N L O}^{(\text {static })}=g_{+}^{2}\left(I_{+}+\Delta I\right)-g_{-}^{2}\left(-I_{-}+\Delta I\right)=\left(-1.03 g_{+}^{2}-0.04 g_{-}^{2}\right) I_{0} .
$$

Thus, the total rescattering contribution is $\left(-0.03 g_{+}^{2}-1.04 g_{-}^{2}\right) I_{0}$, i.e. it is basically negligible for the isoscalar part of the $\pi N$ interaction and it almost equals to the static term for the isovector one. However, this particular cancellation seems to be specific to $\pi d$ scattering and the result might be different for exchange mesons heavier than the pion.

As one might expect intuitively, Pauli principle arguments do not apply to the static piece, for it describes the instantanous exchange of a pion that does

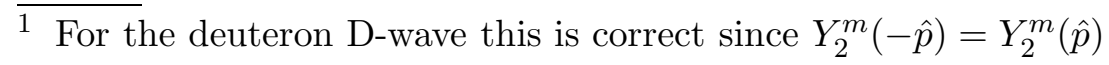


not allow for any two-nucleon intermediate state. However, one comment is necessary at this place. It might be that there is still a potentially sizable cancellation between the terms proportional to $g_{-}^{2}$ in $a^{(1-b o d y)}$ and $a_{L O}^{(\text {static })}$, simply because these terms are of different signs. However, the goal of the present study is to properly isolate the one-body piece from the rescattering piece - this is the precondition to, for example, extract neutron amplitudes from deuteron observables. The isoscalar $\pi N$ scattering length, however, is proportional to the full expression given in Eq. (7) and not just its first term.

\section{Discussion}

As mentioned above, in reality the isovector $\pi N$ interaction is significantly stronger than the isoscalar one in the near threshold regime. Therefore, for this case we may drop all terms proportional to $g_{+}^{2}$ and find, that indeed the static approximation is justified to calculate the rescattering corrections.

The same kind of selection rules as discussed here for $\pi d$ scattering obviously also apply to other meson-nucleus scattering reactions. The first example we will discuss here is coherent $\pi^{0}$ photo- and electroproduction on the deuteron. For this reaction the reasoning is basically identical. Although the leading $\gamma \pi \bar{N} N$ vertex of Kroll-Rudermann type ${ }^{2}$ contains both a spin and an isospin operator and thus can lead to the ${ }^{1} S_{0}$ intermediate $N N$ state, the rescattering vertices again are of the type given in Eq. (1). Based on this observation in Ref. [4] it was concluded that the Pauli principle leads to a vanishing of all rescattering contributions. However, given the discussion at the end of the previous section, we now understand that this conclusion is based on an improper separation of one-body and rescattering terms and indeed a static pion exchange is a good approximation for the leading rescattering contribution. Therefore the quite elaborate calculations of neutral pion photo- and electroproduction based on chiral perturbation theory with static nucleons $[15,16]$ give accurate results.

However, the picture changes when we look at incoherent pion production reactions like $\gamma d \rightarrow \pi^{+} n n$ and for this reaction large effects from the recoils are to be expected. In this context it is interesting to note that the existing data for this reaction are well described by the one-body terms alone, however, the inclusion of rescattering contributions, estimated in the static approximation, lead to a visible deviation of the calculation from the data (see the review [17] and references therein). A possible solution to this is that the recoil corrections strongly diminish the rescattering piece, as discussed above, leaving the one-

2 This refers, of course, to the rescattering corrections since the Kroll-Rudermann vertex only leads to charged pion production. 
body piece as a good approximation to the full result. We will check this conjecture by an explicit calculation in a subsequent publication.

Another case of interest is $\eta$-nucleus scattering. For example in a recent analysis of the world data set on $p d \rightarrow \eta^{3}$ He indications were found that the imaginary part of the $\eta^{3} \mathrm{He}$ scattering length might be significantly smaller than three times the imaginary part of the elementary $\eta N$ amplitude [18], contrary to what is expected from many multiple scattering approaches. Given the findings of above, this should no longer come as a surprise: the ${ }^{3} \mathrm{He}$ wave function is dominated by a quasi-deuteron together with an additional proton in an $s$-wave [19]. In addition, the dominant piece of the $\eta N \rightarrow \pi N$ transition amplitude close to the $\eta$-threshold is spin and momentum-independent, for it is driven by the resonance $S_{11}(1535)$, but is isospin-dependent. Consequently, all the reasoning given above applies and to a good approximation the imarinary parts that originate from the one-body amplitudes have to be cancelled by those from the rescattering and therefore there is no connection between the imaginary part of the $\eta^{3} \mathrm{He}$ scattering length and that for $\eta N$ scattering.

\section{Summary}

In this letter, we have studied the role of recoil corrections in low-energy meson-nucleus reactions. In a simplified model of $\pi d$ scattering, we have shown how to separate the one-body contribution, that embodies the pertient information on the elementary pion-nucleon amplitude, from the rescattering (twobody) corrections, paying particular attention to the constraints from the Pauli principle. Our results are opposite to those of Ref. [4], where it was argued that rescattering contributions are negligible when Pauli forbidden intermediate states occur whereas they are large for Pauli allowed states. The difference was traced to an improper separation of one-body and nuclear contributions in Ref. [4]. Our findings indicate, that, if the S-wave $N N$ intermediate state is Pauli forbidden, the static meson exchange is a good approximation to the full amplitude, whereas in case of the Pauli allowed S-wave intermediate state the (then significant) recoil corrections strongly suppress the nuclear corrections. In this paper the consequences of these insights for other reactions were discussed.

The authors thank A. M. Gasparyan for many interesting discussions. A.E.K. also acknowledges V.G. Ksenzov for useful discussions. This work was partly supported by RFBR grant $\mathrm{N}^{0}$ 02-02-16465 and by 02-02-04001 DFG-RFBR grant 436 RUS 113/652. 


\section{References}

[1] S. Weinberg, Phys. Lett. B 295 (1992) 114.

[2] S. R. Beane, V. Bernard, T.-S. H. Lee and U.-G. Meißner, Phys. Rev. C 57 (1998) 424.

[3] S. R. Beane, V. Bernard, E. Epelbaum, U.-G. Meißner and D. R. Phillips, Nucl. Phys. A 720 (2003) 399.

[4] M. P. Rekalo and E. Tomasi-Gustafsson, Phys. Rev. C 66 (2002) 015203.

[5] V. Baru and A. E. Kudryavtsev, Phys. Atom. Nucl. 60 (1997) 1475

[6] V. Baru, A. E. Kudryavtsev and V. E. Tarasov, Yad. Fiz. 67, N4 (2004), in print, arXiv: nucl-th/0301021.

[7] V. M. Kolybasov and V. G. Ksenzov, Zh. Eksp. Teor. Fiz. 71 (1976) 13; V. G. Ksenzov, Yad. Fiz. 28 (1978) 1249; O. D. Dalkarov, V. M. Kolybasov and V. G. Ksenzov, Nucl. Phys. A 397 (1983) 498.

[8] G. Fäldt, Phys. Scripta 16, 81 (1977)

[9] T. E. O. Ericson and W. Weise, "Pions And Nuclei," Oxford, UK: Clarendon (1988).

[10] S. Weinberg, Phys. Rev. Lett. 17 (1966) 616.

[11] Y. Tomozawa, Nuovo Cim. 46A (1966) 707.

[12] V. M. Kolybasov and A. E. Kudryavtsev, Nucl. Phys. B 41 (1972) 510.

[13] V. Bernard, N. Kaiser and U.-G. Meißner, Int. J. Mod. Phys. E 4 (1995) 193.

[14] R. Machleidt, K. Holinde and C. Elster, Phys. Rept. 149 (1987) 1.

[15] S. R. Beane, V. Bernard, T.-S. H. Lee, U.-G. Meißner and U. van Kolck, Nucl. Phys. A 618 (1997) 381.

[16] H. Krebs, V. Bernard and U.-G. Meißner, Nucl. Phys. A 713 (2003) 405.

[17] J. M. Laget, Phys. Rept. 69 (1981) 1.

[18] A. Sibirtsev, J. Haidenbauer, C. Hanhart and J. A. Niskanen, arXiv: nucl-th/0310079.

[19] V. Baru, J. Haidenbauer, C. Hanhart and J. A. Niskanen, Eur. Phys. J. A 16 (2003) 437. 